\title{
Perception of limb orientation in the vertical plane depends on center of mass rather than inertial eigenvectors
}

\author{
Rolf van de Langenberg • Idsart Kingma • \\ Peter J. Beek
}

Received: 22 November 2005/ Accepted: 5 January 2007/Published online: 7 March 2007

(C) Springer-Verlag 2007

\begin{abstract}
We performed two experiments to test the hypothesis that the perception of limb orientation depends on inertial eigenvectors $\left(\mathbf{e}_{\mathbf{i}}\right)$ against the alternative hypothesis that it depends on the center of mass vector (CM). Whereas $\mathbf{e}_{\mathbf{i}}$ constrains the dynamic torques involved in angular rotation, $\mathbf{C M}$ constrains the static torque necessary to keep the limb aloft in the gravitational field. Hence, possible effects of $\mathbf{e}_{\mathbf{i}}$ and $\mathbf{C M}$ on kinesthetic judgments must be related to the dynamic and static torques, respectively, involved in moving and positioning a limb. In the first experiment, blindfolded participants matched, with upper arms supported, the orientation of their forearms while the forearms' $\mathbf{e}_{\mathbf{i}}$ and $\mathbf{C M}$ were manipulated relative to the elbow. The manipulation of the vector $\mathbf{C M}$ alone induced a matching bias, as did the combined manipulation of $\mathbf{e}_{\mathbf{i}}$ and $\mathbf{C M}$, whereas the manipulation of $\mathbf{e}_{\mathbf{i}}$ alone did not. In the second experiment, participants positioned their unseen and unsupported right arm at an indicated spatial configuration while $\mathbf{e}_{\mathbf{i}}$ and $\mathbf{C M}$ of the right forearm were manipulated as in Experiment 1. As in the first experiment, forearm positioning was affected by the independent manipulation of $\mathbf{C M}$ and the combined manipulation of $\mathbf{e}_{\mathbf{i}}$ and $\mathbf{C M}$, but not by the independent variation of $\mathbf{e}_{\mathbf{i}}$.
\end{abstract}

This research was supported in part by The Netherlands Organization for Scientific Research (NWO) Grant 402-01-040.

R. van de Langenberg $(\bowtie) \cdot$ I. Kingma · P. J. Beek

Faculty of Human Movement Sciences, Vrije Universiteit,

Amsterdam, Van der Boechorststraat 9,

1081 BT Amsterdam, The Netherlands

e-mail: r.vdlangenberg@fbw.vu.nl

R. van de Langenberg · I. Kingma · P. J. Beek Institute for Fundamental and Clinical Human Movement Sciences, Amsterdam, Nijmegen, The Netherlands
Moreover, none of the manipulations affected upper arm positioning. These results refute the claim that the perception of limb orientation (in the vertical plane) is based on $\mathbf{e}_{\mathbf{i}}$ and demonstrate, for the first time, the implication of a limb segment's $\mathbf{C M}$ in the perception of its orientation.

Keywords Limb position sense $\cdot$ Kinesthesis . Proprioception · Information - Center of mass · Inertial eigenvectors - Gravitational torque

\section{Introduction}

How do we perceive where our limbs are in space without having to look at them continuously? Although this question is fundamental to the understanding of both perception and motor control, it is far from resolved. Investigations of the neurophysiological basis of kinesthesis have produced many relevant findings, but they have also highlighted the need for psychophysical concepts that pertain to the role of mechanical, in particular kinetic, information in kinesthetic experiences, as is illustrated by a brief overview of the pertinent literature.

Given that the relative position of limb segments is specific to a particular set of joint angles, the neurophysiological basis of kinesthesis has been sought primarily in neural signals providing geometric information related to joint angles. In principle, such signals may come from mechanoreceptors in joint, skin, and muscle. The role of joint receptors proved to be modest at best as they were found to be mostly silent in the mid-range of motion and to fire only at the extremes of a joint's movement range (e.g., Burgess and Clark 1969; Clark and Burgess 1975; Grigg and Greenspan 1977). Similarly, no prominent role of cutaneous receptors in signaling joint angles was 
established (Gandevia 1996), leaving muscle receptors as the primary candidates for signaling limb geometry. Although skeletal muscles contain both Golgi tendon organs and muscle spindles, only the latter are currently thought to reliably signal muscle length and, hence, inform about limb geometry (Kandel et al. 2000). Goodwin and colleagues firmly established this view by showing that tendon vibrations targeted at primary and secondary spindle endings induced marked illusory joint displacements (e.g., Goodwin et al. 1972a, b). More recently, Ribot-Ciscar et al. (2003) showed that the exclusive reliance on primary muscle spindle afferents may in theory lead to the accurate perception of joint angle.

It is thus beyond doubt that geometrical information pertaining to joint angles, primarily signaled by muscle spindles, plays an important role in kinesthesis. Yet it has become apparent that our sense of limb position and movement is not based exclusively on geometric information. Signals of a kinetic nature (i.e., relating to force or effort), which presumably cannot be conveyed by muscle spindles, probably provide positional information as well. Rymer and D'Almeida (1980) demonstrated such kinetic influences by showing that errors in perceived finger orientation occur upon the generation of isometric contractions. They attributed this effect to a central mechanism receiving muscle force information from Golgi tendon organs. Results of Worringham and Stelmach (1985) suggested that limb kinesthesis depends on gravitational torque $\left(\mathrm{N}_{\mathrm{g}}\right)$, which would also imply that it has a kinetic component. The significance of information related to muscular force or effort was amplified further by recent findings indicating a kinesthetic effect of muscular effort (Proske et al. 2004; Walsh et al. 2004; Winter et al. 2005), motor outflow signals (Gandevia et al. 2006), and muscle activity (Prud'homme and Kalaska 1994).

Given the accumulating evidence that signals related to muscular force or effort are involved in kinesthesis, one may wonder how information relevant to kinesthetis is extracted from such kinetic signals. This issue is far from trivial because, unlike muscle spindle activity, tendon organ activity or motor outflow corrolaries bear no direct relation to muscle length or joint angle. This fact may have contributed to the now widespread view, put forward by McCloskey (1981), that motor outflow corollaries are only used to filter out spindle discharges related to changes in muscle activity so as to obtain accurate muscle length information (see Gandevia 1996). Yet it is possible that both afferent and efferent kinetic signals contribute to kinesthesis independent of muscle spindle activity. Information about muscular force directly reflects the dynamic and static torques involved in actively moving or positioning a limb. Through Newton's laws of motion, those torques are linked to the limb's mass distribution in space.
Therefore, a promising psychophysical approach may be to postulate that an independent kinetic foundation of kinesthesis resides directly in specific characteristics of the limb's mass distribution.

Taking such an approach, Pagano and colleagues (e.g., Pagano and Turvey 1995; Pagano et al. 1996; Garrett et al. 1998) hypothesized that the perception of a limb's spatial orientation depends on its inertial eigenvectors $\left(\mathbf{e}_{\mathbf{i}}\right)$, which represent a characteristic of a limb's mass distribution related to the direction in which it resists rotation (see the appendix for a more detailed explanation of the physical meaning of inertial eigenvectors). Relative to a given point in space, any rigid object has three orthogonal eigenvectors: $\mathbf{e}_{1}, \mathbf{e}_{2}$, and $\mathbf{e}_{3}$. For a forearm rotating around the elbow, $\mathbf{e}_{\mathbf{1}}$ and $\mathbf{e}_{2}$ describe a plane through the elbow, orthogonal to the forearm's longitudinal axis. The axis of minimal resistance against rotation, $\mathbf{e}_{3}$, roughly coincides with the forearm's longitudinal axis. The possible kinesthetic role of $\mathbf{e}_{\mathbf{i}}$ in general and $\mathbf{e}_{3}$ in particular resides in the fact that they reflect the forearm's spatial orientation. In studying limb kinesthesis, it is essential to recognize that information about $\mathbf{e}_{\mathbf{i}}$ is only available when a perceiver actively rotates his or her forearm. After all, $\mathbf{e}_{\mathbf{i}}$ is related to the resistance against rotation and thus exclusively affects the dynamic torques involved in rotation. When a forearm is held stationary, the required muscular torque only has a static component and is therefore independent of $\mathbf{e}_{\mathbf{i}}$.

The so-called inertial eigenvector hypothesis was introduced by Pagano and Turvey (1995) and corroborated in numerous subsequent studies (Pagano et al. 1996; Garrett et al. 1998; Pagano and Turvey 1998; Turvey 1998; Pagano 2000; Riley and Turvey 2001; Riley and Pagano 2003; Bernardin et al. 2005; Riley et al. 2005). All pertinent experiments adopted a similar method in which a single load was attached to the limb at a distance from its longitudinal axis, thereby breaking its coincidence with $\mathbf{e}_{3}$. With the exception of a recent study by Craig and Bourdin (2002, but see Riley and Pagano 2003; Riley et al. 2005) it was found that the perception of limb orientation was biased towards $\mathbf{e}_{3}$, which was taken as evidence for the hypothesis in question. The studies by Pagano et al. (1996) and Garrett et al. (1998) are of particular interest for the present study because they specifically addressed the perception of limb orientation in a vertical (i.e., gravitational) plane and found that it was affected by the aforementioned manipulation of $\mathbf{e}_{\mathbf{3}}$.

However, close scrutiny of this manipulation reveals that an alternative explanation is possible. The placement of a single load off the forearm's longitudinal axis not only introduces a rotation of the vector $\mathbf{e}_{3}$ relative to the elbow, but also displaces the forearm's center of mass. Similar to $\mathbf{e}_{3}$, we view the center of mass as a vector originating at the 
elbow (CM), and its displacement as a rotation of this vector. Importantly, $\mathbf{C M}$ and $\mathbf{e}_{\mathbf{3}}$ constitute principally different characteristics of the forearm's mass distribution. Whereas $\mathbf{e}_{\mathbf{i}}$ exclusively affects the dynamic torques involved in limb rotation, the vector $\mathbf{C M}$ exclusively affects the static gravitational torque $\left(\mathrm{N}_{\mathrm{g}}\right)$. A dependence of kinesthesis on $\mathbf{C M}$ would thus imply a fundamentally different kinetic basis than a dependence on $\mathbf{e}_{\mathbf{i}}$. Yet the possibility that $\mathbf{C M}$, rather than $\mathbf{e}_{3}$, governed the results of Pagano et al. (1996) and Garrett et al. (1998) has never been tested experimentally. In order to disentangle the effects of both variables, and thus to test the inertial eigenvector hypothesis against the center of mass hypothesis, $\mathbf{e}_{\mathbf{i}}$ and $\mathbf{C M}$ must be varied independently. We accomplished such a manipulation and experimental test in the experiments reported below.

\section{Experiment 1}

In the first experiment, we tested the inertial eigenvector hypothesis against the center of mass hypothesis using a forearm-matching task similar to that employed by Pagano et al. (1996) and Garrett et al. (1998). The inertial eigenvector hypothesis predicts that variation in $\mathbf{e}_{\mathbf{3}}$ is sufficient to induce a matching bias. Hence, according to this hypothesis a matching bias should occur in all conditions in which $\mathbf{e}_{3}$ is manipulated. According to the center of mass hypothesis, a pointing bias should occur upon rotation of the vector $\mathbf{C M}$, irrespective of the orientation of $\mathbf{e}_{3}$. In the present experiment, the mass distribution of the forearms was manipulated such that $\mathbf{e}_{\mathbf{3}}$ and $\mathbf{C M}$ either varied independently or covaried (as was the case in the experiments of Pagano and colleagues), thus allowing for a critical test of the two hypotheses of interest.

\section{Method}

Twenty healthy participants (11 female and 9 male; all right-handed; mean age 28.5 years, SD 6.0 years) participated voluntarily in the experiment. They were not familiar with the type of experiment or the rationale behind it. The experiment, which was conducted in accordance with the 1964 Declaration of Helsinki, was approved formally by the ethical committee of our faculty and carried out with the adequate understanding and written informed consent of all participants.

Participants were blindfolded and sat on a stool with their upper arms resting on a wooden surface. They were to match the orientation of their unseen forearms, to which carbon fiber frames with two brass loads were fixed in order to achieve the desired orientations of $\mathbf{e}_{3}$ and $\mathbf{C M}$ (see Fig. 1).

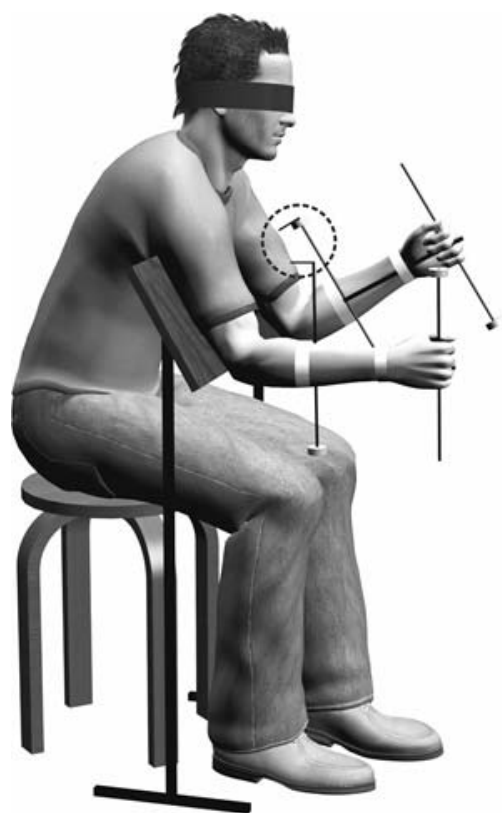

Fig. 1 The experimental setup of Experiment 1. Participants were blindfolded and sat on a stool with their upper arms resting on a wooden surface oriented at a $60^{\circ}$ angle with the horizontal. Participants' armpits touched the upper edge of the wooden surface and their upper arms were positioned in parallel and flush with the wooden surface. The carbon fiber frames, used to attach loads to the forearms (see text for a detailed description), were to remain in parallel vertical planes while forearm orientation was matched by flexion and extension of the elbows. Two straws with a length of $5 \mathrm{~cm}$ extended from the upper end of the proximal crosspieces, preventing the loads from touching the upper arms and marking the maximal allowed elbow flexion. The straws are visible inside the dotted circle

Each carbon fiber frame consisted of a stem, $30 \mathrm{~cm}$ in length and $1 \mathrm{~cm}$ in diameter, and two parallel crosspieces, both $40 \mathrm{~cm}$ in length and $0.6 \mathrm{~cm}$ in diameter, that were pierced through the stem at a $90^{\circ}$ angle. These crosspieces were placed $22 \mathrm{~cm}$ apart with the most distal crosspiece at $4 \mathrm{~cm}$ from the distal end of the stem. The total mass of each frame was $32 \mathrm{~g}$. The frames were fixed to the ventral side of the forearm, along its longitudinal axis, and their distal tips protruded from the closed hand between the ring finger and the middle finger. The crosspieces were positioned at a distance of 11 and $33 \mathrm{~cm}$, respectively, from the medial epicondyl of the humerus. A thin straw with a length of $5 \mathrm{~cm}$ protruded from the upper part of the proximal crosspiece (see Fig. 1), preventing the upper arm from contacting the loads. Moreover, by touching the upper arm, it signaled the smallest elbow angle allowed in the experiment (approximately $80^{\circ}$ ).

Forearm matching occurred in eight experimental conditions and a control condition. In the experimental conditions, two cylindrical brass loads were attached to the frames to achieve the desired orientations of $\mathbf{e}_{\mathbf{3}}$ and $\mathbf{C M}$. The experimental manipulations are illustrated in Fig. 2 

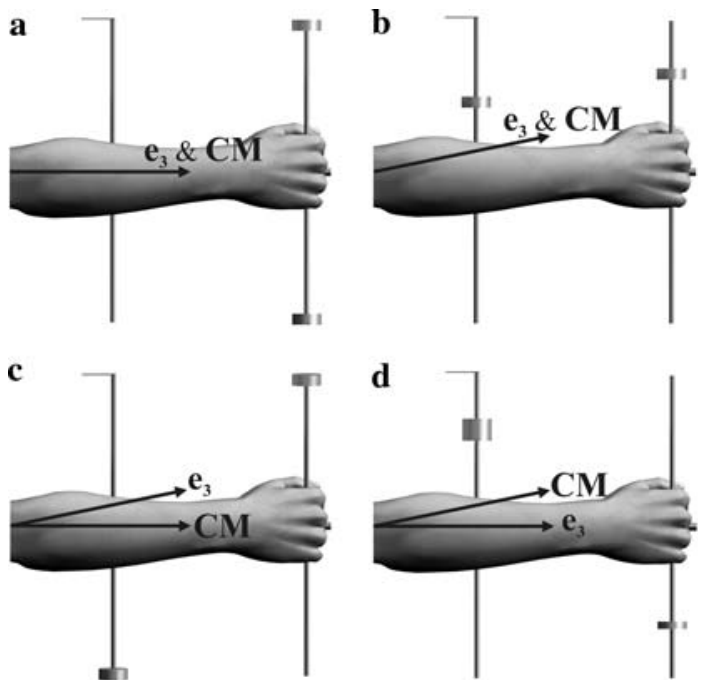

Fig. 2 The experimental manipulations of the vectors $\mathbf{e}_{\mathbf{3}}$ and $\mathbf{C M}$. $\mathbf{C M}$ is the vector from the elbow joint to the average position of the (loaded) forearm's mass (i.e., the effective point of origin of gravitational force). The eigenvector $\mathbf{e}_{3}$ is the axis through the elbow joint about which the (loaded) forearm's rotational inertia is minimal. Panel a shows the loads as they were attached to one of the forearms in conditions 7 and 8 (in which the other forearm remained unloaded). The loads are equal in mass, are placed at equal but opposite distances from the forearm's longitudinal axis, and have equal distances to elbow. They thus do not induce a rotation of either $\mathbf{C M}$ or $\mathbf{e}_{3}$ relative to an unloaded arm. In panel $\mathrm{b}$ (conditions 5 and 6), the asymmetrical load placement causes both vectors $\mathbf{e}_{\mathbf{3}}$ and $\mathbf{C M}$ to be rotated towards the loads. In panel $\mathbf{c}$ (conditions 1 and 2), the loads have equal mass and are placed at equal but opposite distances from the forearm's longitudinal axis, so that no rotation of $\mathbf{C M}$ is induced. Yet the loads have different distances to the elbow, so that they do induce a rotation of $\mathbf{e}_{3}$. Panel $\mathbf{c}$ thus represents the independent variation of $\mathbf{e}_{\mathbf{3}}$. Finally, in panel $\mathbf{d}$ (conditions 3 and 4), the two loads are placed equidistant from the forearm's longitudinal axis and have different distances to the elbow, as in panel c, but now their masses differ with the heaviest load being closest to the elbow. This leads to $\mathbf{C M}$ being rotated towards the greatest load, while $\mathbf{e}_{3}$ still coincides with the longitudinal axis. Panel $\mathbf{d}$ thus represents the independent variation of $\mathbf{C M}$

and the exact masses and positions of the loads in the eight experimental conditions are reported in Table 1. The magnitude and direction of $\mathbf{e}_{3}$ rotation induced by the loads was calculated by adding the loads' inertia tensors relative to the elbow to that of the unloaded forearm (using the parallel axis theorem) and subsequently calculating the new orientation of $\mathbf{e}_{\mathbf{1}}, \mathbf{e}_{2}$, and $\mathbf{e}_{\mathbf{3}}$ by diagonalizing the resulting tensor. In conditions 1 and $2, \mathbf{e}_{3}$ was thus manipulated $5^{\circ}$ toward flexion in one arm (the right arm in condition 1 ; the left arm in condition 2 ) and $5^{\circ}$ toward extension in the other, without manipulating $\mathbf{C M}$, resulting in a $10^{\circ}$ difference between the eigenvectors of the forearms. In conditions 3 and $4, \mathbf{C M}$ was manipulated $5^{\circ}$ toward flexion in one arm (the right arm in condition 3; the left arm in condition 4) and $5^{\circ}$ toward extension in the other, without manipulating $\mathbf{e}_{3}$, resulting in a $10^{\circ} \mathbf{C M}$ difference between the forearms. In conditions 5 and 6 , both $\mathbf{e}_{3}$ and $\mathbf{C M}$ were manipulated such that there was a $10^{\circ} \mathbf{e}_{\mathbf{3}}$ and $\mathbf{C M}$ difference between both forearms. In conditions 7 and 8, one arm remained unloaded (the left arm in condition 7; the right arm in condition 8) and loads were attached to the other arm in a symmetrical way, i.e., without inducing a rotation of either $\mathbf{e}_{\mathbf{3}}$ or $\mathbf{C M}$. A ninth condition, in which both forearms remained unloaded, served as control condition. The maximal value of $\mathrm{N}_{\mathrm{g}}$ about the elbow was equal in both arms in all conditions, except for conditions 7 and 8 , which were included to explicitly test for the effect of $\mathrm{N}_{\mathrm{g}}$ suggested by Worringham and Stelmach (1985). In conditions 1 through 6, the placement of the loads merely induced an asymmetrical mass distribution relative to the forearm's longitudinal axis.

Participants performed the nine conditions in nine corresponding trial blocks. Before each trial block, they assumed a position in which one arm was flexed at an elbow angle of $80^{\circ}$, which was achieved by letting the tip of the straw just touch the upper arm, and the other arm was fully extended. Note that, in this starting position, a perceptual reference was ensured both in the flexed arm (by the straw) and in the fully extended arm (by the end of the elbow's movement range), preventing any drift effects over trials. From this position, one of the forearms (the target arm) was moved towards the other forearm until the experimenter called out "stop". The experimenter ensured that this stop signal was given at a different arm orientation in each trial. The other arm (the matching arm) was then moved towards the target arm until the participant perceived the orientation of the two forearms to be identical. At this moment, the participant stopped the movement and called out "ja" ("yes"). After registering the orientation of both forearms (see below), the experimenter instructed the participant to assume the starting position for the next trial: The arm that was flexed at the start of the previous trial was now extended and vice versa. The target arm was alternately the left and the right arm. Participants were instructed to keep both arms and the crosspieces they enclosed with their hands in a vertical (i.e., sagittal) plane at all times.

Each trial block was started with either the left or the right arm extended, and with either the extended or the flexed arm as the target arm. The four resulting starting configurations were counterbalanced across participants, with each individual participant starting all trial blocks from the same assigned configuration. Each trial block consisted of two series of eight matching trials. In one series the extended arm was the target arm and in the other the flexed arm was the target arm. All participants performed 144 matching trials in total (9 trial blocks; 16 trials per block). The duration of an experimental session was approximately $45 \mathrm{~min}$. 
Table 1 Masses and positions of the loads and corresponding rotations of $\mathbf{e}_{\mathbf{3}}$ and $\mathbf{C M}$ in the experimental conditions of experiment 1

\begin{tabular}{|c|c|c|c|c|c|c|c|c|c|c|}
\hline \multirow[b]{3}{*}{ Condition } & \multicolumn{4}{|c|}{ Right forearm } & \multicolumn{4}{|c|}{ Left forearm } & \multirow[b]{3}{*}{$\Delta \mathbf{e}_{3}^{\mathrm{b}}$} & \multirow[b]{3}{*}{$\Delta \mathbf{C M}^{\mathrm{c}}$} \\
\hline & \multicolumn{2}{|l|}{ Load 1} & \multicolumn{2}{|l|}{ Load 2} & \multicolumn{2}{|l|}{ Load 1} & \multicolumn{2}{|l|}{ Load 2} & & \\
\hline & Mass (g) & $x, y^{\mathrm{a}}(\mathrm{cm})$ & Mass (g) & $x, y(\mathrm{~cm})$ & Mass (g) & $x, y(\mathrm{~cm})$ & Mass (g) & $x, y(\mathrm{~cm})$ & & \\
\hline 1 & 150 & $11,-18.8$ & 150 & $33,18.8$ & 150 & $11,18.8$ & 150 & $33,-18.8$ & $10^{\circ}$ & $0^{\circ}$ \\
\hline 2 & 150 & $11,18.8$ & 150 & $33,-18.8$ & 150 & $11,-18.8$ & 150 & $33,18.8$ & $-10^{\circ}$ & $0^{\circ}$ \\
\hline 3 & 300 & $11,13.8$ & 100 & $33,-13.8$ & 300 & $11,-13.8$ & 100 & $33,13.8$ & $0^{\circ}$ & $10^{\circ}$ \\
\hline 4 & 300 & $11,-13.8$ & 100 & $33,13.8$ & 300 & $11,13.8$ & 100 & $33,-13.8$ & $0^{\circ}$ & $-10^{\circ}$ \\
\hline 5 & 150 & $11,5.8$ & 150 & $33,12.8$ & 150 & $11,-5.8$ & 150 & $33,-12.8$ & $10^{\circ}$ & $10^{\circ}$ \\
\hline 6 & 150 & $11,-5.8$ & 150 & $33,-12.8$ & 150 & $11,5.8$ & 150 & $33,12.8$ & $-10^{\circ}$ & $-10^{\circ}$ \\
\hline 7 & 100 & $33,18.8$ & 100 & $33,-18.8$ & - & - & - & - & $0^{\circ}$ & $0^{\circ}$ \\
\hline 8 & - & - & - & - & 100 & $33,18.8$ & 100 & $33,-18.8$ & $0^{\circ}$ & $0^{\circ}$ \\
\hline
\end{tabular}

${ }^{a} x$ refers to the distance of the load's center of mass from the elbow along the arm's longitudinal axis; $y$ refers to the orthogonal distance of the load's center of mass from the arm's longitudinal axis, where a positive (negative) distance indicates a position on the radial (ulnar) side of the forearm

${ }^{\mathrm{b}} \Delta \mathbf{e}_{3}$ indicates the angle between the smallest eigenvector $\left(\mathbf{e}_{3}\right)$ of the left and the right forearm. A positive angle indicates that $\mathbf{e}_{3}$ of the right forearm was manipulated towards elbow flexion and $\mathbf{e}_{3}$ of the left forearm towards elbow extension

c $\Delta \mathbf{C M}$ indicates the angle between the center of mass vector $(\mathbf{C M})$ of the left and the right forearm. A positive angle indicates that $\mathbf{C M}$ of the right forearm was oriented towards elbow flexion and $\mathbf{C M}$ of the left forearm towards elbow extension

Forearm orientation was measured using a $3 \mathrm{D}$ active movement registration system (Optotrak 3020, Northern Digital Inc., Waterloo, Canada), which was calibrated using a coordinate frame with one axis aligned with the gravitational vertical, and one parallel to the horizontal axis described by participants' elbow and shoulder joints. During the experiment, the position of four infrared markers was registered: two markers on the distal tip of each frame stem, and two markers on the horizontal axes described by the two elbow joints and the two shoulder joints, respectively. The latter two markers were placed on an adjustable carbon fiber frame to the left of the participants after they assumed the correct starting position. In this starting position, both armpits contacted the upper edge of the wooden board, which ensured that the elbow joints as well as the shoulder joints described a horizontal axis. The experimenter registered the position of the four markers each time a participant indicated that the orientation of the forearms was matched. For each participant and for each trial, the angle of the two elbow joints, projected onto a sagittal plane, was calculated from the position data of the four Optotrak markers. The elbow angles of both arms were subsequently averaged to obtain the angle around which matching occurred in each trial. To obtain the direction and magnitude of matching errors, the elbow angle of the right arm was subtracted from that of the left arm. A positive matching error thus indicated that elbow angle was smallest in the right arm, that is, that the right arm had a flexion bias relative to the left arm. Finally, matching errors in the control condition were subtracted from those in each experimental condition to obtain matching biases due to the eight experimental manipulations. We first examined the range of elbow angles around which matching occurred in each condition, the pattern of matching errors within conditions, and the matching errors in the control condition. Subsequently, we analyzed the matching errors according to a repeated measures analysis of variance (ANOVA) with condition (9 levels) and repetition (16 levels) as within-subject factors. Finally, we tested the matching biases due to the eight experimental manipulations using one-sample two-tailed $t$ tests.

\section{Results and discussion}

The average and the range of the elbow angles around which matching occurred in each of the nine conditions was $115.5^{\circ}$ (SD over conditions $1.0^{\circ}$ ) and $26.9^{\circ}$ (SD over conditions $1.3^{\circ}$ ), respectively. The low standard deviations indicate that both the average and the range of matching angles only differed marginally between conditions.

Before turning to the effects of our experimental manipulations on the matching errors, we first examined matching errors within conditions. Participants showed a significant overshoot of the target arm's orientation with the matching arm $\left(t(19)=2.41, P=0.027, \eta^{2}=0.23\right)$, which is in keeping with Worringham and Stelmach (1985), but not with Pagano et al. (1996) who did not find such an effect. Matching errors of a representative participant in the control condition are shown in Fig. 3. The alternating pattern of errors in this figure is a manifestation of the aforementioned overshoot effect. After all, the left and the right arm alternatingly assumed a flexed starting 


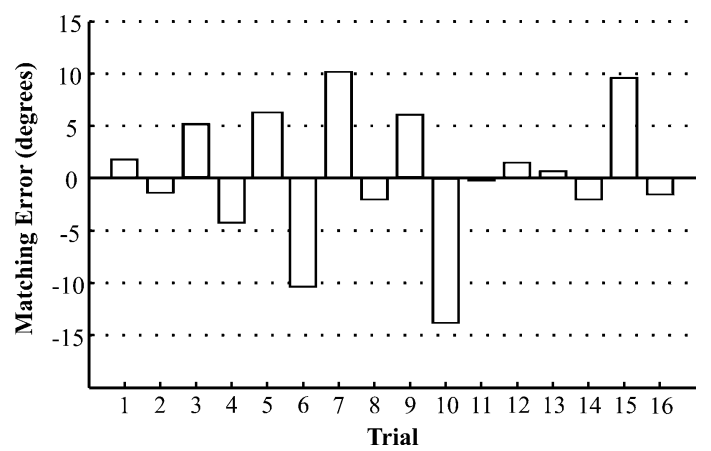

Fig. 3 Matching errors of a representative participant in the control condition of Experiment 1, in which no loads were attached to the forearms. The alternating pattern of errors over trials reflects a tendency to overshoot the target arm with the matching arm

position, so that an overshoot of the target arm would alternatingly lead to a positive and a negative matching bias. In this way, matching biases due to overshoot canceled out after averaging over trials. Accordingly, in the control condition, matching errors did not deviate significantly from zero $(P=0.53)$ after averaging over trials (mean $0.5^{\circ}$; SD across participants $3.7^{\circ}$ ). However, partly due to a tendency to overshoot the target arm, error variability across trials was considerable (SD 5.7; see Fig. 3). This trial-to-trial variability is comparable to that reported by Soechting (1982).

The ANOVA performed on the matching errors in all conditions revealed that they were affected significantly by condition $\left(F(8,152)=10.5, P<0.001, \eta_{\mathrm{p}}^{2}=0.36\right)$, but neither by repetition $(P=0.80)$ nor by the condition $\times$ repetition interaction $(P=0.46)$. Figure 4 shows the average matching biases (with $95 \%$ confidence intervals) due to the eight experimental manipulations. No significant effect of $\mathbf{e}_{3}$ was found $(P=0.72$ and $P=0.69$, in conditions 1 and 2, respectively). In contrast, the $10^{\circ}$ difference in $\mathbf{C M}$ orientation resulted in significant matching biases of $-1.5^{\circ}$ in condition $3\left(t(19)=-2.92, P=0.009, \eta^{2}=0.31\right)$ and of $2.2^{\circ}$ in condition $4(t(19)=2.70, P=0.014$, $\left.\eta^{2}=0.28\right)$. The combined manipulations of $\mathbf{e}_{3}$ and $\mathbf{C M}$ had similar effects: A $10^{\circ}$ difference of $\mathbf{e}_{3}$ and $\mathbf{C M}$ orientation between the arms resulted in significant matching biases of $-1.9^{\circ}$ in condition $5\left(t(19)=-3.63, P=0.002, \eta^{2}=0.41\right)$ and of $2.5^{\circ}$ in condition $6(t(19)=4.13, P=0.001$, $\eta^{2}=0.47$ ). A negative (positive) matching bias implied that the flexion of the right arm relative to the left arm was smaller (greater) than in the control condition. The direction of the significant effects of conditions 3 through 6 was consistent with the hypothesis that perceived orientation would be biased towards CM. Finally, loading one of the arms symmetrically (i.e., without rotating either $\mathbf{e}_{3}$ or $\mathbf{C M}$ ) while leaving the other arm unloaded did not induce significant matching biases relative to the control condition

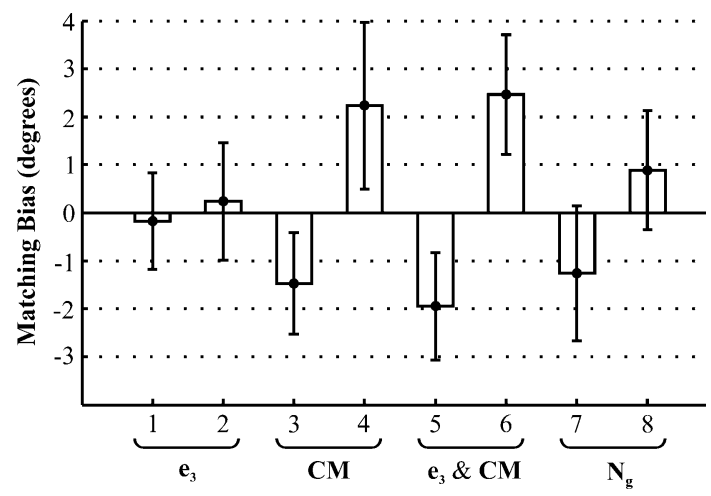

Fig. 4 Average matching biases relative to the control condition in the different manipulation conditions of Experiment 1. A positive bias implies that the flexion of the right arm relative to the left arm was greater in that manipulation condition than in the control condition. Error bars show the $95 \%$ confidence interval of the matching biases. Only $\mathbf{e}_{3}$ was rotated in conditions 1 and 2, only $\mathbf{C M}$ was rotated in conditions 3 , and 4 , and $\mathbf{e}_{\mathbf{3}}$ and $\mathbf{C M}$ were rotated together in conditions 5 and 6 . In conditions 1,3 , and 5 , rotations were toward flexion in the right arm and toward extension in the left arm (and vice versa in conditions 2,4 , and 6). In conditions 7 and $8, \mathbf{e}_{\mathbf{3}}$ and $\mathbf{C M}$ were not rotated; only $\mathrm{N}_{\mathrm{g}}$ differed between the two forearms: in condition 7 (8), it was greater in the right (left) arm

$\left(t(19)=-1.87, \quad P=0.077, \quad \eta^{2}=0.16\right.$ for condition 7 ; $t(19)=1.50, P=0.15, \eta^{2}=0.11$ for condition 8 ), although there was a trend towards an extension bias in the loaded arm (see Fig. 4). Such an extension bias would be consistent with the direction of the effect of $\mathrm{N}_{\mathrm{g}}$ (or muscular effort) reported in the literature (Worringham and Stelmach 1985; Proske et al. 2004; Walsh et al. 2004; Winter et al. 2005). More importantly, the significant effect of $\mathbf{C M}$ in conditions 3 and 4, together with the absence of a significant effect of $\mathbf{e}_{3}$ in conditions 1 and 2, indicates that the results of Pagano et al. (1996) and Garrett et al. (1998) should be interpreted in retrospect as a sensitivity to $\mathbf{C M}$ rather than $\mathbf{e}_{\mathbf{i}}$.

Given that people can perceive the orientation of their limbs in space, and not only relative to each other, one may wonder whether the present results, favoring the center of mass hypothesis, generalize to the perception of the orientation of an unsupported arm in extrinsic space. In this situation, loading the forearm not only affects the torques around the elbow joint, as was the case in the present experiment, but also the torques around the shoulder joint. In principle, this could restrict or alter the implication of $\mathbf{C M}$ in the perception of limb orientation. These possibilities were investigated in Experiment 2.

\section{Experiment 2}

In the second experiment, we manipulated $\mathbf{e}_{3}$ and/or $\mathbf{C M}$ of the forearm in the same manner as in Experiment 1, but this 
time to test their effects on the perception of the orientation of an unsupported arm in a vertical plane. Based on the results of Experiment 1, we hypothesized that perceived forearm orientation would be affected by $\mathbf{C M}$ and not by $\mathbf{e}_{3}$. We further hypothesized that the manipulations would not affect the perceived orientation of the upper arm, even though we recognized that loading the forearm does affect the torque at the shoulder when the upper arm is not supported. The latter hypothesis was motivated from the insight that the shoulder torque can only be informative about the orientation of the upper arm when the elbow torque is taken into account as well. We reasoned that manipulation of the forearm's CM would induce torques at the elbow indicating an altered forearm orientation. Given that the corresponding change in the torque pattern at the shoulder is consistent with this change in forearm orientation, we further reasoned that it too would indicate an altered forearm orientation, rather than an altered upper arm orientation.

\section{Method}

Twenty healthy participants (13 female and 7 male; all right-handed; mean age 26.0 years, SD 4.3 years) participated voluntarily in the experiment. They were not familiar with the type of experiment or the rationale behind it. The experiment, which was conducted in accordance with the 1964 Declaration of Helsinki, was approved formally by the ethical committee of our faculty and carried out with the adequate understanding and written informed consent of all participants.

The experimental setup is shown in Fig. 5. Participants were seated on a stool besides a vertical wooden board. Their right arm and shoulder were placed through a circular hole in the board between two overlapping pieces of lycra cloth that prevented them from looking through the hole to the other side of the board. Participants were wearing a Tshirt, of which the right sleeve was rolled up to just above the acromion to allow the placement of a marker on the shoulder (see below). The stool was adjusted such that the projection of the forearm on the vertical plane could coincide with line segments 1 through 3 , and that of the upper arm with line segment 4 . The experimental task consisted of matching the orientation of the unseen forearm with line segment 1,2 , or 3 , while maintaining the upper arm parallel to line segment 4 . Line segments 1, 2, and 3 were oriented at angles of $15^{\circ}, 0^{\circ}$, and $-15^{\circ}$, respectively, relative to horizontal. Line segment 4 was oriented at an angle of $-60^{\circ}$ relative to horizontal, which corresponded to the orientation of the upper arm support in Experiment 1. Brass weights were attached to the forearm by means of a carbon fiber frame-as in Experiment 1-to achieve the desired orientations of the vectors $\mathbf{e}_{3}$ and $\mathbf{C M}$ (see Fig. 2).

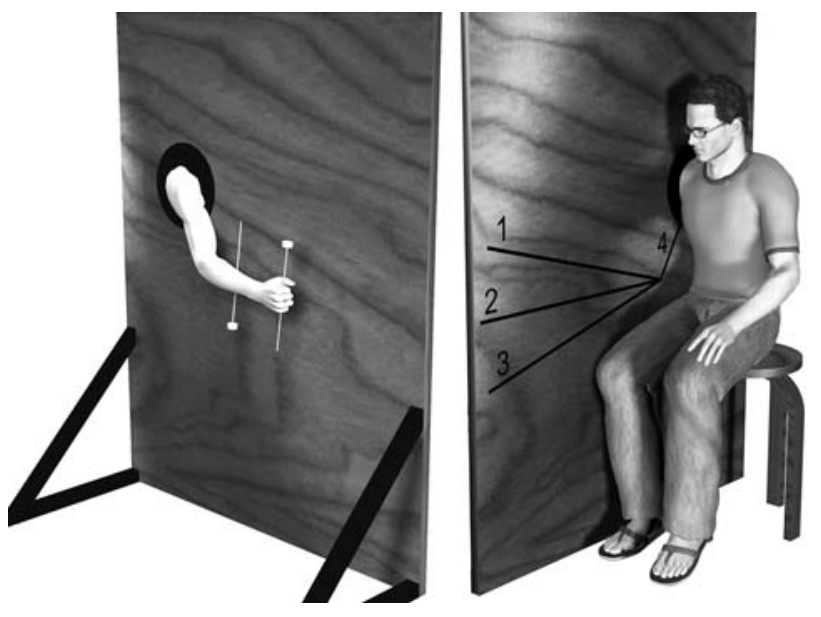

Fig. 5 The experimental setup of Experiment 2. Participants sat on a stool besides a vertical wooden board. Their right arm and shoulder were placed through a circular hole in the board between two overlapping pieces of lycra cloth. Participants were wearing a T-shirt, of which the right sleeve was rolled up to uncover the shoulder. The stool was adjusted such that the projection of the right forearm on the vertical plane could coincide with line segments 1,2 and $3\left(15^{\circ}, 0^{\circ}\right.$ and $-15^{\circ}$ relative to horizontal, respectively) and the projection of the upper arm with line segment $4\left(-60^{\circ}\right.$ relative to horizontal). The experimental task consisted of matching the orientation of the unseen forearm alternately with line segments 1,2 and 3 , while maintaining the unsupported upper arm parallel to line segment 4 . The carbon fiber frame, used to attach loads to the forearm (see text for a detailed description), was to remain parallel to the board at all times

The straw, used in Experiment 1 to prevent arm orientations smaller than about $80^{\circ}$, was not used in the present experiment because the risk that loads would contact the upper arm was much smaller than in Experiment 1.

Arm positioning was required in seven experimental conditions and a control condition. Load placements in the experimental conditions were equal to those in experiment 1. The exact masses and positions of the loads can thus be found in the five leftmost columns of Table 1 (i.e., those referring to the right arm). In conditions 1 and $2, \mathbf{e}_{\mathbf{3}}$ was independently manipulated by $5^{\circ}$ towards extension and $5^{\circ}$ towards flexion, respectively, resulting in a $10^{\circ}$ difference in $\mathbf{e}_{3}$ orientation between the two conditions. A $10^{\circ}$ difference in $\mathbf{C M}$ orientation was achieved between conditions 3 and 4 , and a $10^{\circ}$ difference in the orientation of both $\mathbf{e}_{3}$ and $\mathbf{C M}$ was achieved between conditions 5 and 6 . In condition 7, loads were attached to the forearm in a symmetrical way, i.e., without inducing a rotation of either $\mathbf{e}_{3}$ or CM. An eighth condition, in which the forearm remained unloaded, served as control condition.

The eight conditions were performed in eight corresponding trial blocks. Before each trial block, participants assumed a position in which the upper arm was oriented parallel to line segment 4 , and the arm was either fully flexed or fully extended (counterbalanced across partici- 
pants). The experimenter then called out one of the numbers 1 through 3 to indicate the target line segment for the forearm, upon which the participant rotated his or her forearm around the elbow towards the target line segment. Participants were instructed to stop moving the arm and push a button with the left hand when they perceived their forearm to be parallel to the target line segment and their upper arm to be parallel to line segment 4 . If the arm was initially fully flexed (extended), participants subsequently fully extended (flexed) their arm. The experimenter then called out a new target line segment and the forearm was again rotated about the elbow (albeit in opposite direction) to match the orientation of the indicated line segment. Participants thus alternated between full elbow flexion and full elbow extension, and assumed the instructed arm configuration as a pause in each elbow flexion and extension movement. They were instructed to keep the crosspieces they enclosed with their right hand parallel to the wooden board at all times. In each trial block, participants matched the orientation of their forearm with each of the three target line segments four times, resulting in a total of 12 trials per block. Throughout each trial block, the upper arm was to remain parallel to line segment 4 . All participants thus performed a total of 96 matching trials ( 8 trial blocks; 12 trials per block). The duration of an experimental session was approximately $30 \mathrm{~min}$.

The configuration of the arm was measured using Optotrak. The position of six infrared markers was continuously registered at $100 \mathrm{~Hz}$ during each trial block: a marker on the tip of the carbon fiber frame (marker 1), a marker on either side on the distal crosspiece (markers 2 and 3), a marker on the lateral epicondyl of the humerus (marker 4), a marker just below the acromion on the deltoid muscle (marker 5), and finally a marker (marker 6) connected to the button in participants' left hand. The latter marker only emitted infrared light when the button was pushed. For each participant and for each trial, the orientation of the upper arm and forearm was calculated from the position data of markers 1,4 , and 5 . The target orientations of forearm $\left(15^{\circ}, 0^{\circ}\right.$, or $-15^{\circ}$ relative to horizontal) and upper arm $\left(-60^{\circ}\right.$ relative to horizontal) were subtracted from the actual orientations that forearm and upper arm had at each moment the button was pushed. Errors in forearm and upper arm orientation were analyzed according to a repeated measures analysis of variance (ANOVA). For forearm orientation errors, we analyzed condition (8 levels), target (3 levels, corresponding to line segments 1, 2, and 3; see Fig. 5), and repetition (4 levels) as within-subject factors. For errors in upper arm orientation, we analyzed only the factors condition and repetition. The difference in positioning errors between conditions 1 and 2 (e $\mathbf{e}_{3}$ manipulation), 3 and 4 (CM manipulation), 5 and 6 (combined $\mathbf{e}_{3}$ and $\mathbf{C M}$ manipulation), and 7 and $8\left(\mathrm{~N}_{\mathrm{g}}\right.$ manipulation without $\mathbf{e}_{\mathbf{3}}$ and $\mathbf{C M}$ rotation), collapsed over targets, were subsequently compared using paired-samples two-tailed $t$ tests. Note that in the present experiment four $t$ tests rather than eight (as in Experiment 1) sufficed, because only one arm was manipulated rather than two.

\section{Results and discussion}

The average error in aligning the forearm with line segments 1 , 2, and 3 was $4.0^{\circ}$ in upward direction. The ANOVA performed on these errors revealed that they were affected significantly by condition $(F(7,133)=8.4$, $\left.P<0.001, \quad \eta_{\mathrm{p}}^{2}=0.31\right)$ and target $(F(2,38)=7.0$, $\left.P=0.003, \eta_{\mathrm{p}}^{2}=0.27\right)$, but not by repetition $(P=0.15)$. The target effect indicated that a lower line segment was associated with smaller upward forearm positioning errors than a higher line segment. None of the two-way interactions was significant (all $P$ 's $>0.5$ ), whereas the three-way interaction just reached significance $(F(42,798)=1.4$, $\left.P=0.042, \eta_{\mathrm{p}}^{2}=0.07\right)$. As the latter effect had a marginal effect size and had no readily apparent origin, we abstained from seeking an account for it. The results of the paired $t$ tests performed on forearm positioning errors are shown in Fig. 6. No significant effect of adding mass in a symmetrical configuration was found $(P=0.18)$. The effect of $\mathbf{e}_{3}$ was also non-significant $(P=0.08)$. In contrast, $\mathbf{C M}$ rotation significantly affected forearm orientation $(t(19)=4.1$, $P=0.001, \eta^{2}=0.47$ ), as did rotation of $\mathbf{e}_{3}$ and $\mathbf{C M}$

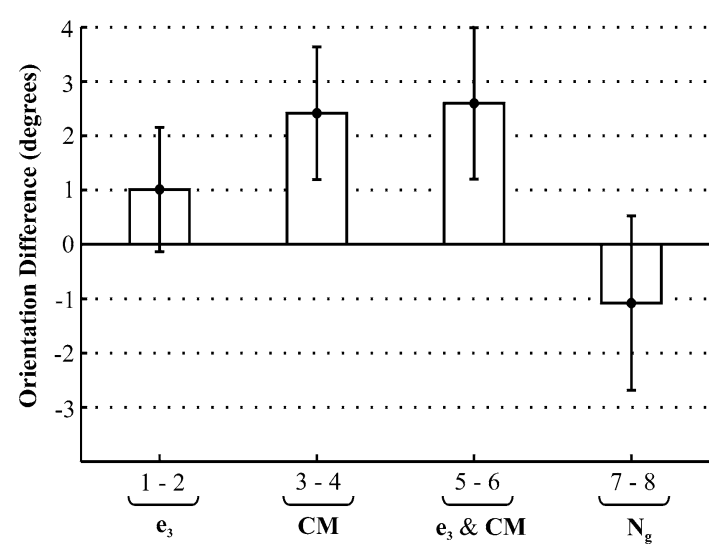

Fig. 6 Difference in forearm orientation between conditions 1 and 2 (e $\mathbf{e}_{3}$ manipulation), 3 and 4 (CM manipulation), 5 and 6 (combined $\mathbf{e}_{3}$ and $\mathbf{C M}$ manipulation), and 7 and $8\left(\mathrm{~N}_{\mathrm{g}}\right.$ manipulation) in Experiment 2. In conditions 1,3 and 5 (2, 4 and 6), $\mathbf{e}_{\mathbf{3}}$ and/or $\mathbf{C M}$ were manipulated downward (upward). In condition 7, in which loads were attached to the frame symmetrically, $\mathrm{N}_{\mathrm{g}}$ was larger than in condition 8 , in which no loads were attached to the frame. For $\mathbf{e}_{3}$ and/or $\mathbf{C M}$ manipulation (three leftmost bars), a positive difference indicates that downward manipulation was associated with a more upward forearm orientation. For $\mathrm{N}_{\mathrm{g}}$ manipulation (rightmost bar), a negative difference indicates that a greater $\mathrm{N}_{\mathrm{g}}$ was associated with a more downward forearm orientation. Error bars show the 95\% confidence interval of the orientation differences 
together $\left(t(19)=3.9, P=0.001, \eta^{2}=0.44\right)$. A $10^{\circ}$ difference of $\mathbf{C M}$ orientation alone was accompanied by a $2.4^{\circ}$ difference in forearm orientation. A $10^{\circ}$ difference of both $\mathbf{e}_{3}$ and $\mathbf{C M}$ orientation corresponded to a $2.6^{\circ}$ difference in forearm orientation. As expected, a more downward (upward) orientation of the forearm's CM always accompanied a more upward (downward) forearm orientation.

The average error in aligning the upper arm with line segment 4 was $7.4^{\circ}$ in downward direction. The ANOVA performed on these errors revealed that they were only significantly affected by repetition $(F(11,209)=2.4$, $\left.P=0.008, \eta_{\mathrm{p}}^{2}=0.11\right)$. The main effect of condition and the condition $\times$ repetition interaction just failed to reach significance $(P=0.056$ and $P=0.093$, respectively). The paired $t$ tests revealed that neither $\mathbf{C M}$ rotation nor $\mathbf{e}_{3}$ rotation significantly affected upper arm orientation errors (both $P$ 's $>0.7$ ). The effect of symmetrical mass addition was also non-significant $(P>0.3)$. Finally, no effect of the combined $\mathbf{e}_{3}$ and $\mathbf{C M}$ manipulation was revealed, albeit that this effect approached significance $(P=0.071)$.

In sum, forearm orientation was affected in a very similar way as in the matching task of Experiment 1, whereas the unsupported upper arm was not affected, or only marginally at best, by the manipulation of $\mathbf{e}_{\mathbf{3}}$ and/or CM. The results thus corroborate both hypotheses forwarded in the introduction to the present experiment.

\section{General discussion}

With the overarching aim to uncover the kinetic foundation of kinesthesis, we tested the inertial eigenvector hypothesis against the alternative hypothesis that the perception of limb orientation in the vertical plane depends on CM. To this end, two experiments were conducted, one in which the orientations of the forearms had to be matched (Experiment 1) and one in which the orientation of a single arm had to be matched to an external reference configuration (Experiment 2). The results of both experiments supported the center of mass hypothesis and were inconsistent with the inertial eigenvector hypothesis. Experiment 1 revealed that the results of Pagano et al. (1996) and Garrett et al. (1998) should be interpreted in retrospect as a sensitivity to $\mathbf{C M}$ rather than $\mathbf{e}_{\mathbf{i}}$. Experiment 2 generalized the results of Experiment 1 for forearm matching with the upper arm supported to orienting a single, unsupported limb with respect to extrinsic space. In particular, the results of Experiment 2 indicated that forearm manipulation in a vertical plane shifted perceived forearm orientation in that plane without causing a significant shift in the perceived orientation of the upper arm. The combined results of Experiments 1 and 2 raise the crucial question how $\mathbf{C M}$ might mediate limb kinesthesis. We address this question at two levels, first extensively in terms of the torques involved and then briefly and more tentatively in terms of possible neurophysiological mechanisms.

In both experiments, placing mass symmetrically (see Fig. 2a) had no significant effect on the perception of forearm orientation, but did show a trend towards an extension bias in the heavier arm. It may be that adding mass symmetrically only had a small effect, which could explain the inconsistency of previous findings in this regard. In particular, whereas the results of Worringham and Stelmach (1985) indicate an effect of symmetrical mass placement, Soechting (1982) as well as Darling and Hondzinski (1999) failed to find such an effect. More important for the present discussion is the suggestion in the latter studies that $\mathrm{N}_{\mathrm{g}}$ does not play a significant role in limb kinesthesis. This suggestion stands in stark contrast with the marked effects of $\mathbf{C M}$ found in the present experiments, which necessarily imply that $\mathrm{N}_{\mathrm{g}}$ plays a role in limb kinesthesis, albeit in a different manner than considered previously. After all, as we already noted in the introduction, $\mathrm{N}_{\mathrm{g}}$ is the only detectable variable affected by $\mathbf{C M}$ rotation. So how does $\mathbf{C M}$ affect $\mathrm{N}_{\mathrm{g}}$, and how is this different from the $\mathrm{N}_{\mathrm{g}}$ manipulation in previous studies and our conditions with symmetrical mass addition? In an unloaded arm, $\mathrm{N}_{\mathrm{g}}$ is a sinusoid function of arm inclination that reaches its maximum at a horizontal arm orientation and becomes zero when the arm is vertical (see solid gray curve in Fig. 7). Adding loads symmetrically (as shown in Fig. 2a) may be viewed as a scaling of this relationship. That is, it increases $\mathrm{N}_{\mathrm{g}}$ at each inclination angle by a constant factor so that the maximum (minimum) of $\mathrm{N}_{\mathrm{g}}$ still occurs at a horizontal (vertical) arm orientation (see the dotted black curve in Fig. 7). Yet a rotation of $\mathbf{C M}$ by asymmetric mass addition (as shown in Fig. 2b, d) not only scales but also horizontally shifts this relationship, so that the maximum of $\mathrm{N}_{\mathrm{g}}$ no longer occurs at a horizontal arm orientation (see the solid black curve in Fig. 7). Hence, this horizontal shift reflects the rotation of the vector CM. One may therefore conclude that $\mathrm{N}_{\mathrm{g}}$ plays an indirect role in the perception of limb orientation, namely as a mediator of $\mathbf{C M}$ orientation, rather than $\mathrm{N}_{\mathrm{g}}$ magnitude itself being informative about limb orientation.

In a vertical plane, the perception of limb orientation relative to extrinsic coordinates (e.g., the gravitational vertical) has often been found to be more accurate than perception with respect to intrinsic coordinates (e.g., joint angle, or the trunk- and head-longitudinal axes; Soechting 1982; Worringham and Stelmach 1985; Worringham et al. 1987; Darling 1991; Darling and Hondzinski 1999). It follows from the present results that the use of extrinsic coordinates in limb kinesthesis may substantially benefit from CM-related information, conveyed by a horizontal 


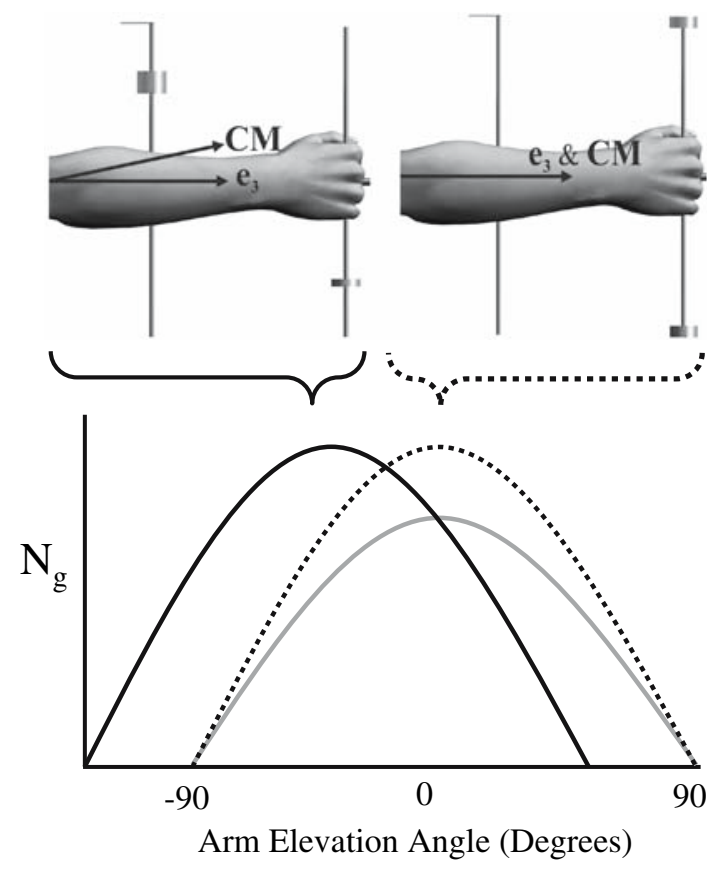

Fig. 7 Schematic depiction of the relationship between $\mathrm{N}_{\mathrm{g}}$ and arm inclination angle (i.e., the angle of the arm's longitudinal axis with the horizontal) in an unloaded arm (gray solid curve), a symmetrically loaded arm (black dotted curve) and an asymetrically loaded arm in which CM is rotated (black solid curve). Whereas symmetrical mass addition only changes the scaling of the relationship, CM rotation also induces a horizontal shift. We propose that the latter shift governs the sensitivity to $\mathbf{C M}$ demonstrated in the present study

shift of $\mathrm{N}_{\mathrm{g}}$ as a function of arm inclination, which could explain this higher accuracy in extrinsic coordinates. The issue of extrinsic versus intrinsic coordinate systems is also a relevant dimension when comparing the center of mass hypothesis with the inertial eigenvector hypothesis.

Unlike $\mathbf{C M}, \mathbf{e}_{\mathbf{i}}$ cannot aid the perception of limb orientation in extrinsic, earth-fixed coordinates. After all, as we noted in the introduction, the possible perceptual role of $\mathbf{e}_{\mathbf{i}}$ resides exclusively in its effect on the dynamic torques involved in limb rotation, which are independent of a limb's orientation in extrinsic space. Hence, the perception of limb orientation through the detection of inertial eigenvectors necessarily implies an intrinsic coordinate system, similar to that involved in the detection of muscle length or joint angle. This important point was overlooked by Garrett et al. (1998), who found that their manipulation of $\mathbf{e}_{\mathbf{i}}$ (and implicitly $\mathbf{C M}$ ) affected the perception of forearm orientation relative to the gravitational vertical and interpreted this as an effect of $\mathbf{e}_{\mathbf{i}}$. It follows from the preceding argument that this interpretation was invalid because $\mathbf{e}_{\mathbf{i}}$, being gravity independent, cannot convey such information. Hence, irrespective of the present empirical evidence, Garrett et al. (1998) could have attributed their findings to gravity-dependent information.
Because the experimental tasks in the present study allow for the use of extrinsic as well as intrinsic axes, the apparent dominance of $\mathbf{C M}$ over $\mathbf{e}_{\mathbf{i}}$ cannot be explained in terms of their respective coordinate systems. So what did underlie the difference in perceptual effect between $\mathbf{e}_{\mathbf{i}}$ and $\mathbf{C M}$ in the present experiments? The answer may reside in their respective signal-to-noise ratios, or saliences (cf. van de Langenberg et al. 2007). Recall that information about $\mathbf{e}_{\mathbf{i}}$ is only reflected in the dynamic torques involved in active limb movement. In the present experiments, as well as in Pagano et al. (1996) and Garrett et al. (1998), the arms were moved at a relatively low angular velocity, implying that the contribution of dynamic torques to the muscular tension was small relative to the static torque $\mathrm{N}_{\mathrm{g}}$. It follows that the salience of $\mathbf{e}_{\mathbf{i}}$ must have been low relative to that of CM, which exclusively affects $\mathrm{N}_{\mathrm{g}}$. In general, one can say that during slow movements or stationary postures, the salience of $\mathbf{C M}$ will be superior to that of $\mathbf{e}_{\mathbf{i}}$. During fast movements, the salience of $\mathbf{e}_{\mathbf{i}}$ will increase at the cost of the salience of $\mathbf{C M}$, which leaves open the possibility that the perception of arm orientation in fast movements, at least in intrinsic coordinates, is affected by $\mathbf{e}_{\mathbf{i}}$ as well.

The results of Experiment 2 generalized the role of $\mathbf{C M}$ from matching the orientation of contralateral forearms to orienting a single arm in extrinsic space. They further showed that the perception of upper arm orientation was unaffected by the manipulation of the forearm's $\mathbf{C M}$, even though the manipulation affected $\mathrm{N}_{\mathrm{g}}$ at both the elbow and the shoulder due to the absence of an upper arm support. The latter finding may be explained in two ways: Participants may have exploited $\mathrm{N}_{\mathrm{g}}$ at the shoulder to obtain information about the configuration of the whole arm in space, as we anticipated in the introduction to Experiment 2 , or they may have discounted it, only taking $\mathrm{N}_{\mathrm{g}}$ into account at the elbow. The latter alternative would suggest that the role of $\mathbf{C M}$ in the perception of limb orientation pertains only to the most distal rigid segment, whereas the former alternative would suggest that its role is more general, supporting the perception of the orientation of both proximal and distal limb segments. This issue may be resolved in experiments in which the center of mass vectors of both distal and proximal limb segments (e.g., both the forearm and the upper arm) are manipulated in tasks similar to that used in Experiment 2.

In seeking an encompassing account of the perception of limb orientation, it is important to note that we only tested the effect of $\mathbf{C M}$ and $\mathbf{e}_{3}$ manipulations in the vertical plane. Manipulation of $\mathbf{C M}$ in the horizontal plane would only affect the direction of $\mathrm{N}_{\mathrm{g}}$, not its magnitude, and hence cannot be mediated by the aforementioned horizontal shift in the relationship between arm orientation and $\mathrm{N}_{\mathrm{g}}$ magnitude (see Fig. 7). In a recent study (van de Langenberg et al. 2007), we tested the effect of horizontal $\mathbf{C M}$ and $\mathbf{e}_{\mathbf{3}}$ 
manipulations in a pointing task similar to that adopted in Experiments 1 and 2 of Pagano and Turvey (1995). We found essentially the same pattern of results as in the present study: Perceived arm orientation was affected by the horizontal manipulation of $\mathbf{C M}$ but not by that of $\mathbf{e}_{\mathbf{3}}$. It thus appears that humans are sensitive to changes in both torque magnitude and direction, and hence to $\mathbf{C M}$ rotations in $3 \mathrm{D}$.

Another important point of discussion is that the observed perceptual biases were consistently smaller than the magnitude of $\mathbf{C M}$ rotation, as was the case in previous studies on the effect of vertical (CM and) $\mathbf{e}_{\mathbf{3}}$ rotations (Pagano et al. 1996; Garrett et al. 1998). However, the relative sizes of our effects (i.e., 15-26\% of the actual CM rotation) were smaller than those observed in those previous studies (i.e., $40-45 \%$ of the actual $\mathbf{C M}$ rotation). We suspect that this difference is related to differences in the magnitude of $\mathbf{C M}$ manipulation, which was $10^{\circ}$ in our Experiment 1 and only about $5^{\circ}$ in Pagano et al. (1996) and Garrett et al. (1998). In a study on the effect of horizontal $\mathbf{e}_{3}$ (and $\mathbf{C M}$ ) rotations, Bernardin et al. (2005) adopted three manipulation magnitudes in a single experiment and indeed found a strong negative relationship between effect size and manipulation magnitude (upon an increase of $\mathbf{C M}$ manipulation from about $1.3^{\circ}$ to about $5.5^{\circ}$ effect size decreased from 38 to $13 \%$ ). In addition to manipulation magnitude, other factors, such as manipulation direction (i.e. horizontal or vertical) and exploration style, may also influence the magnitude of CM's effect on the perception of limb orientation. These factors should be explored further in future research. Regardless of their precise contributions, however, one can already conclude that the perceptual biases introduced by manipulating $\mathbf{C M}$ and $\mathbf{e}_{\mathbf{3}}$ are in general incomplete. It follows from this general observation that additional information, unrelated to either $\mathbf{C M}$ or $\mathbf{e}_{3}$, must have been employed. The use of information unrelated to $\mathbf{C M}$ is also apparent in conditions in which the arm's CM cannot be detected, such as when the limb is moved passively to a certain position (see e.g., Lee et al. 2003; Ulkar et al. 2004) or when gravity is absent (see e.g., Lackner and DiZio 2000).

Notwithstanding the preceding qualifications, the present findings clearly underscore that there is a kinetic component to limb kinesthesis, which has important implications for its possible neural basis. More specifically, the apparent dependence of limb kinesthesis on $\mathbf{C M}$ points to an important role of neural signals related to muscular torque or effort. As noted in the introduction, several other psychophysical studies provided support for this view (Rymer and D'Almeida 1980; Prud'homme and Kalaska 1994; Proske et al. 2004; Walsh et al. 2004; Winter et al. 2005; Gandevia et al. 2006). Although these studies clearly challenge the view that limb kinesthesis relies on muscle spindle activity alone (see Ribot-Ciscar et al. 2003), they do not discard the possibility that signals related to muscular torque or effort only serve to accurately interpret spindle discharges in terms of muscle length changes, as proposed by McCloskey (1981, see also Gandevia 1996). The effect of $\mathbf{C M}$ on limb kinesthesis indicates that, in addition to such an indirect role, kinetic signals may convey information about the distribution of a limb's mass in space and hence directly affect kinesthesis, independent of geometric information about muscle length or joint angle. Golgi tendon organs seem particularly suited for fulfilling this function, given that their activity is closely associated with muscle force (Kandel et al. 2000). This implies that the possible implication of Golgi tendon organs in limb kinesthesis may need to be reconsidered and that a mechanism akin to that suggested by Rymer and d'Almeida (1980) may indeed be possible.

\section{Appendix}

The inertial eigenvectors are reflected in the dynamic torques involved in active limb movement. Notably, a rotation about an axis that coincides with an eigenvector does not require a torque about any axis other than the rotation axis. In the following we will illustrate this conception. Consider a weightless rod with a point-mass $(p)$ attached to one end and fixed at the other end $(O)$. The rod rotates about an axis through $O$ that is orthogonal to the rod's longitudinal axis (illustrated in Fig. 8a). During such a rotation, the pointmass has a centripetal acceleration $\left(\mathbf{a}_{\mathrm{c}}\right)$, which is directed towards $O$. In the case of non-uniform rotation (i.e., rotation with non-zero angular acceleration) it also has a tangential acceleration $\left(\mathbf{a}_{\mathbf{t}}\right)$, tangent to the circular path it describes. At $O, \mathbf{a}_{\mathbf{t}}$ is maintained by a torque $\left(\mathbf{T}_{\mathbf{t}}\right)$ along the axis of rotation. No torque is required to maintain $\mathbf{a}_{\mathrm{c}}$ because it has no lever arm relative to $O$. Now consider a change of the rotation axis from an orthogonal orientation to an orientation of, say, $45^{\circ}$ with the rod's longitudinal axis (Fig. 8b). Importantly, because $\mathbf{a}_{\mathbf{c}}$ is now no longer directed at $O$, and hence does have a lever arm relative to $O$, it involves a torque oriented orthogonal to the rotation axis: the so-called centripetal torque $\left(\mathbf{T}_{\mathrm{c}}\right)$. In the absence of this torque, the required centripetal acceleration of the point-mass cannot be sustained and the rotation axis will revert to the orientation of Fig. $8 \mathrm{a}-\mathrm{a}$ situation in which $\mathbf{T}_{\mathrm{c}}$ is not required. The absence of a centripetal torque orthogonal to the axis of rotation marks a rotation about an inertial eigenvector. Hence, the possible significance of inertial eigenvectors to the problem of limb kinesthesis is that they may be identified by a sensitivity to the centripetal torques involved in moving the limbs, and thus inform about their spatial orientation. 


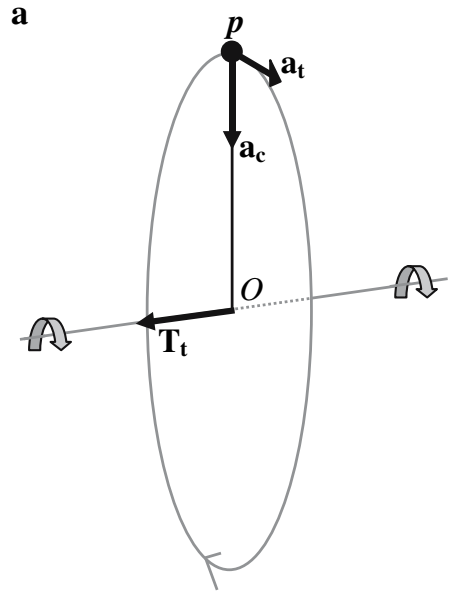

Fig. 8 Illustration of the fact that rotation about an inertial eigenvector only requires a torque about the rotation axis. In each panel, a point-mass $(p)$, fixed to the end of a weightless rod, rotates about an axis through $O$. In panel a, the axis of rotation is orthogonal to the rod's longitudinal axis. This rotation only involves a tangential torque $\left(T_{t}\right)$ along the axis of rotation to generate the tangential

\section{References}

Bernardin D, Isableu B, Fourcade P, Bardy BG (2005) Differential exploitation of the inertia tensor in multi-joint arm reaching. Exp Brain Res 167:487-495

Burgess PR, Clark FJ (1969) Characteristics of knee joint receptors in cat. J Physiol-Lond 203:317-335

Clark FJ, Burgess PR (1975) Slowly adapting receptors in cat kneejoint — can they signal joint angle? J Neurophysiol 38:1448-1463

Craig CM, Bourdin C (2002) Revisited: the inertia tensor as a proprioceptive invariant in humans. Neurosci Lett 317:106-110

Darling WG (1991) Perception of forearm angles in 3-dimensional space. Exp Brain Res 87:445-456

Darling WG, Hondzinski JM (1999) Kinesthetic perceptions of earthand body-fixed axes. Exp Brain Res 126:417-430

Gandevia SC (1996) Kinesthesia: roles for afferent signals and motor commands. In: Rowell LB, Shepherd JT (eds) Handbook of physiology. Section 12. Exercise: regulation and integration of multiple systems. Oxford University Press, New York, pp 128172

Gandevia SC, Smith JL, Crawford M, Proske U, Taylor JL (2006) Motor commands contribute to human position sense. J Physiol (London) 571:703-710

Garrett SR, Pagano CC, Austin G, Turvey MT (1998) Spatial and physical frames of reference in positioning a limb. Percept Psychophys 60:1206-1215

Goodwin GM, McCloskey D, Matthews PB (1972a) Contribution of muscle afferents to kinesthesia shown by vibration induced illusions of movement and by effects of paralyzing joint afferents. Brain 95:705-748

Goodwin GM, McCloskey D, Matthews PB (1972b) Proprioceptive illusions induced by muscle vibration - contribution by musclespindles to perception. Science 175:1382-1384

Grigg P, Greenspan BJ (1977) Response of primate joint afferent neurons to mechanical stimulation of knee-joint. J Neurophysiol 40:1-8

Kandel ER, Schwartz JH, Jessell TM (2000) Principles of neural science. McGraw-Hill, New York b

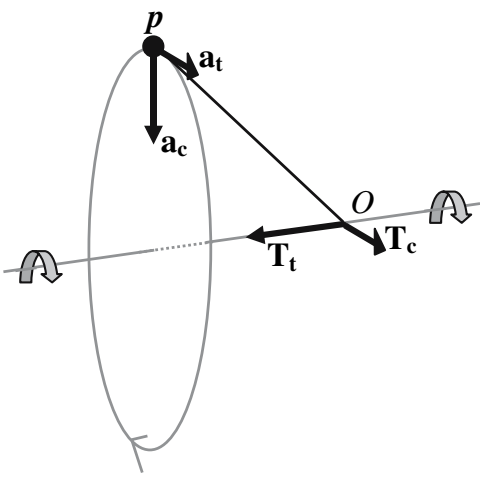

acceleration $\mathrm{a}_{\mathrm{t}}$. In panel $\mathbf{b}$, the axis of rotation is not orthogonal to the rod's longitudinal axis so that $\mathrm{a}_{\mathrm{c}}$ is not directed at $O$. It thus involves a centripetal torque $\left(T_{c}\right)$, oriented orthogonal to the rotation axis. Hence, the rotation axis in panel a represents an eigenvector whereas that in panel $\mathbf{b}$ does not

Lackner JR, DiZio P (2000) Human orientation and movement control in weightless and artificial gravity environments. Exp Brain Res 130:2-26

Lee HM, Liau JJ, Cheng CK, Tan CM, Shih JT (2003) Evaluation of shoulder proprioception following muscle fatigue. Clin Biomech $18: 843-847$

McCloskey D (1981) Corollary discharges: motor commands and perception. In: Brooks V (ed) Handbook of physiology. American Physiological Society, Bethesda, pp 1415-1447

Pagano CC (2000) The role of the inertia tensor in kinesthesis. Crit Rev Biomed Eng 28:1-2

Pagano CC, Garrett SR, Turvey MT (1996) Is limb proprioception a function of the limb's inertial eigenvectors? Ecol Psychol 8:4369

Pagano CC, Turvey MT (1995) The inertia tensor as a basis for the perception of limb orientation. J Exp Psychol Hum Percept Perform 21:1070-1087

Pagano CC, Turvey MT (1998) Eigenvectors of the inertia tensor and perceiving the orientations of limbs and objects. J Appl Biomech 14:331-359

Proske U, Gregory JE, Morgan DL, Percival P, Weerakkody NS, Canny BJ (2004) Force matching errors following eccentric exercise. Hum Movem Sci 23:365-378

Prud'homme MJL, Kalaska JF (1994) Proprioceptive activity in primate primary somatosensory cortex during active arm reaching movements. J Neurophysiol 72:2280-2301

Ribot-Ciscar E, Bergenheim M, Albert F, Roll JP (2003) Proprioceptive population coding of limb position in humans. Exp Brain Res 149:512-519

Riley MA, Pagano CC (2003) Inertial eigenvectors play a role in proprioception: comment on Craig an Bourdin (2002). Ecol Psychol 15:229-240

Riley MA, Shaw TH, Pagano CC (2005) Role of the inertial eigenvectors in proprioception near the limits of arm adduction range of motion. Hum Movem Sci 24:171-183

Riley MA, Turvey MT (2001) Inertial constraints on limb proprioception are independent of visual calibration. J Exp Psychol Hum Percept Perform 27:438-455 
Rymer WZ, D'Almeida A (1980) Joint position sense-the effects of muscle-contraction. Brain 103:1-22

Soechting JF (1982) Does position sense at the elbow reflect a sense of elbow joint angle or one of limb orientation? Brain Res 248:392-395

Turvey MT (1998) Dynamics of effortful touch and interlimb coordination. J Biomech 31:873-882

Ulkar B, Kunduracioglu B, Cetin C, Guner RS (2004) Effect of positioning and bracing on passive position sense of shoulder joint. Br J Sports Med 38:549-552

van de Langenberg R, Kingma I, Beek PJ (2007) Mechanical invariants are implicated in dynamic touch as a function of their salience in the stimulus flow. J Exp Psychol Hum Percept Perform 32:1093-1106 van de Langenberg R, Kingma I, Beek PJ (2006) The perception of limb orientation depends on the center of mass. J Exp Psychol Hum Percept Perform (in press)

Walsh LD, Hesse CW, Morgan DL, Proske U (2004) Human forearm position sense after fatigue of elbow flexor muscles. J Physiol (London) 558:705-715

Winter JA, Allen TJ, Proske U (2005) Muscle spindle signals combine with the sense of effort to indicate limb position. J Physiol (London) 568:1035-1046

Worringham CJ, Stelmach GE (1985) The contribution of gravitational torques to limb position sense. Exp Brain Res 61:3842

Worringham CJ, Stelmach GE, Martin ZE (1987) Limb segment inclination sense in proprioception. Exp Brain Res 66:653-658 